\title{
Localization algorithm of Bluetooth sensor network
}

\author{
Maoxiang $\mathrm{Ji}^{1}$, Yao Yao ${ }^{2,3}$, Chunxia Zhang ${ }^{4}$, Weiyong Jiang ${ }^{5}$, Lei Zhang ${ }^{2,3}$ \\ 1.Key Laboratory of Geography Information, Ministy of education, school of geography science, East China Normal University, \\ Shanghai 200241, China \\ 2. Engineering Center of SHMEC for Space Information and GNSS, East China Normal University, Shanghai 200241, China \\ 3. Shanghai Key Laboratory of Multidimensional Information Processing, East China Normal University, Shanghai 200241, China \\ 4. Shanghai Innovation Center for Beidou Science \& Technology, Shanghai 200083, China \\ 5. Shanghai Zhujiajiao High School, Shanghai 201713, China
}

Abstract: The indoor positioning technology is the most important research field in the location based service (LBS). RFID, ZigBee, Bluetooth and UWB are popular technologies in the indoor positioning system. However, the single technology cannot realize the high accuracy. Bluetooth sensor network continues to advance due to the recent dramatic progress in sensor devices. The innovative use of the received signal strength indicator (RSSI) also yields new applications in human position estimation, an important function in safe and secure services, especially for the elderly, and energy efficiency in small areas or homes. This paper introduces the Bluetooth sensor network which is set in the experiment rooms. It proposes a simple method for estimating human position, together with a new signal processing procedure that uses RSSI. This method is simple and has the exciting benefit of compatibility with existing devices and existing wireless sensor networks, the current RSSI function is employed more effectively by analyzing the statistical properties of the RSSI values in particular environment. Two experiments verify the performance of the proposed method. At last, the constraint algorithm is proposed in three-dimension environment, especially keep the result at some constraining height. And the feasibility and accuracy of the proposed model is checked.

Key words RSSI; Bluetooth; Constraint Algorithm

\section{Introduction}

The demand of acquiring the position of the terminals are becoming increasingly urgent no matter in outdoor or indoor environment. Nowadays, RFID, ZigBee, ultrasonic technology and UWB are popular technologies in indoor positioning systems. The positioning algorithm can be classified into two categories, one based on ranging and one based on signal strength. Methods such as TOA(Time of Arrive), AOA(Angle of Arrive), TDOA(Time Difference of Arrive) belong to the former category. Under indoor environment, the multipath interference and serious reflection affect the accuracy of the distance estimation. However, RSSI technology is more stable when receiving signals so it becomes the hotspot in recent years. One outstanding methods based 
on RSSI is Bluetooth wireless communication technology which works on the frequency band of 2.4GHz. The Bluetooth signals can provide the received signal strength, link quality and transmit power level. These parameters can be adopted to estimate the distances between the terminals and the access points. Furthermore, the constraining algorithm is proposed.

The structure of this paper is as follows: Section I is the introduction of the popular indoor positioning technologies and algorithms. Section II introduces the Bluetooth sensor network and the experiment environment. In this section, the whole Bluetooth sensing process is stated and the concept of Bluetooth sensor network is proposed and introduced.Section III presents the RSSI distance measurement model to verify the feasibility of the Bluetooth indoor positioning. The constraining algorithm is also proposed to keep the terminal positions within some boundaries. Section IV analyzes the precision of the constraining algorithm. Section V is the conclusion part.

\section{Bluetooth sensor network}

\subsection{Wireless technology}

Bluetooth technology is a wireless communication technology, work in the global common 2.4GHz ISM band, Bluetooth technology is applicable for a loose network, Bluetooth technology adopt a scheme which can quickly confirm frequency hopping, this feature makes Bluetooth has good anti-jamming capability and system stability. Bluetooth technology can achieve a small range of wireless connectivity and automatic networking in small network environment. In addition, each Bluetooth device has a unique global 48 bit device address ${ }^{[2-4]}$. Bluetooth network ${ }^{[5]}$ has two distinct characteristics: First, mobility, achieve "anytime, anywhere, free" to networking and access Internet; Second, automatic access, different Bluetooth Can automatically establish the link between each other when devices are limited within prescribed space and prescribed number. In this paper, we use the related control software to achieve sniffers establish the connection and transfer the data without user intervention.

\section{2 network structure}

The entire system use Internet as backbone network for data communication. Terminal network use Bluetooth for data communication. Finally, the system realizes localization.

\subsection{System Components}




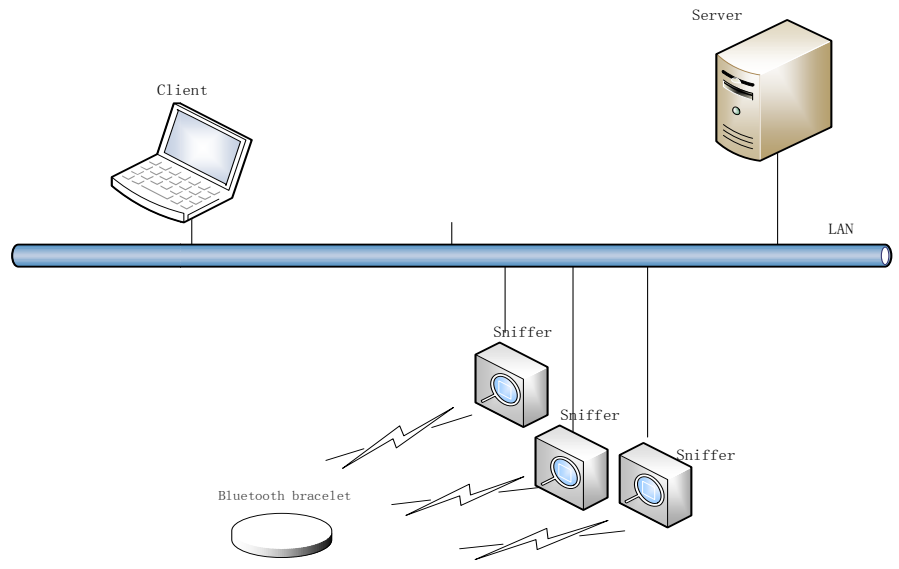

Fig. 1. system architecture

AS is illustrated in Figure 1,Bluetooth sensing system is mainly composed of five parts: Bluetooth bracelet ,Bluetooth sniffer, network infrastructure, data server, client .The function of each kind of equipment is as follows:

1)Bluetooth bracelet

Using a brand of bracelet as a Bluetooth transmitter, Bluetooth bracelet in an acceptable state, and carried by the user.

2)Bluetooth sniffer

Sniffer is mainly used for collecting the surrounding WIFIand Bluetooth BLE information.
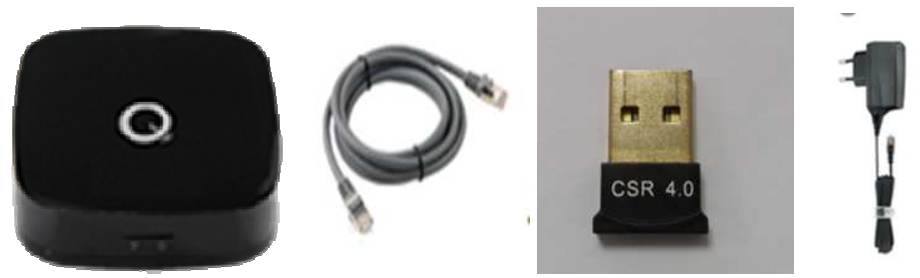

Fig. 2.MB8800X Bluetooth sniffer

3)Infrastructure network

The sniffer can use WIFI or cable access to the Internet. The information of BLE device is uploaded to the remote server and use UDP as transmission's protocol. The data packet consists of the message header, payload, Signature.From the packet we can get the relevant useful information,such as the Mac address of AP or Bluetooth gateway,the number of wireless terminals in one time scanned.The MAC address and RSSI value of a single wireless terminal, etc.

4)Data server

The server is mainly responsible for the storage of information and computing.Finally, through the constraint algorithm for positioning.

5)Client

The client receives location information from data server and displays it on the client. 


\section{RSSI distance measurement model}

The experiment room is equipped with several Bluetooth sniffers, which make up the Bluetooth sensor network. TOA/TDOA and AOA positioning algorithms are difficult to realize in Bluetooth network so RSSI method is adopted. According to the principle of RSSI, there are two main parts of the positioning method: distance measurement and the positioning algorithm. In the distance measurement step, the distance model $\mathrm{f}(\mathrm{RSSI}, \mathrm{d})^{[6,7]}$ is established basing on the observation environment. The distances can be calculated using the RSSI values. It is obvious that the accuracy of distance estimation has a lot to do with the establishment of the model.

The received signal strength indicator theory concludes the attenuation of the signal strength between the receiving and transmitting appliances as an empirical model in order to calculate the distance. However, it is difficult to realize the high-precision positioning because of the time-variant characteristic of RSSI and the unpredictable environment.

In this study, two experiments were conducted in two test rooms respectively in order to get the signal strength in different environment and verify the feasibility of the proposed empirical model. In the test rooms, the Bluetooth sniffer is placed in the area without any block and at the reasonable height. The same terminal communicates with the Bluetooth sniffer at different distances to get the RSSI values. In order to establish the correct model, the statistical properties should be analyzed at first step.

\subsection{Statistical Properties of the RSSI values}

There exists some deviation between the RSSI value of single observation and the calculation value of the distance measurement model due to the environmental factors. However the errors obey some particular statistical properties. From the laws concluded from these properties, the relationship between the distance and RSSI can be found. Furthermore, the constraint algorithm is proposed. Under the experimental scene of this study, three parameters are analyzed in order to find the law: mean value of the signal, signal variance and the skewness of the signals.

Mean value. The mean values of the signals denotes the average distribution of the signal amplitude ${ }^{[8,9,10]}$. Table 3.1 shows the distribution of signal mean values from different distances. In this experiment, ten samples are collected every one meter. The mean value is calculated as follow:

$$
\overline{R S S I}=\frac{1}{n} \sum_{i=1}^{n} R S S I_{i}
$$

By analyzing the trend of the mean values of RSSI signals, it can be concluded that the signal strength presents a logarithmic attenuation as the distances increase as a whole. There appears 
some little fluctuation because of the multipath error and blocks.

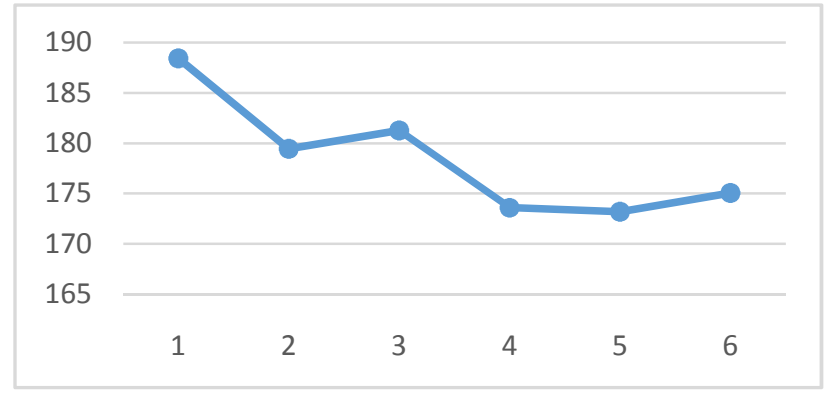

Fig. 3. ResultofMean value

Signal variance: describes the deviation between the observed signal and the signal mean, which indicates the fluctuation of the signals ${ }^{[8,9,10]}$. The larger the variance is, the more instability the signals bear. Signal variance is calculated as:

$$
\sigma^{2}=\frac{1}{n-1} \sum_{i=1}^{n}\left(R S S I_{i}-\overline{R S S I}\right)^{2}
$$

According to the result of the experiment, it can be concluded that the mean variance of 5 different spots is $3.44 \mathrm{dBm}$, the maximum variance is $0.223 \mathrm{dBm}$ while the minimum value is $8.88 \mathrm{dBm}$. In conclusion, the fluctuation of the signal amplitude is inevitable when the distance is larger than six meters. So in the practical positioning process, these points should be abandoned.

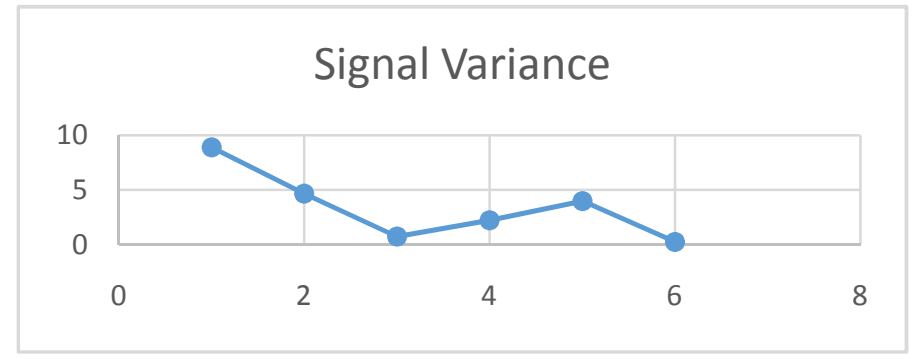

Fig. 4.ResultofSignal variance

Skewness is a concept describing the degree of asymmetry in statistics ${ }^{[1,12,13]}$. This concept is applied in this study to present the deviation of the signal distribution, including the direction and the degree. The function of skewness is presented as follow:

$$
\mathrm{SK}=\frac{\mathrm{n} \sum_{\mathrm{i}=1}^{\mathrm{n}}\left(\mathrm{RSSI}_{\mathrm{i}}-\overline{\mathrm{RSSI}}\right)^{3}}{(\mathrm{n}-1)(\mathrm{n}-2) \sigma^{3}}
$$

According to the function above, the deviation coefficient of the signals at different distances can be acquired. Combined with the logarithmic decrement relationship from the mean values, the average skewness is close to zero and draws near the standard skewness. In conclusion, the distribution is symmetrical approximately. 


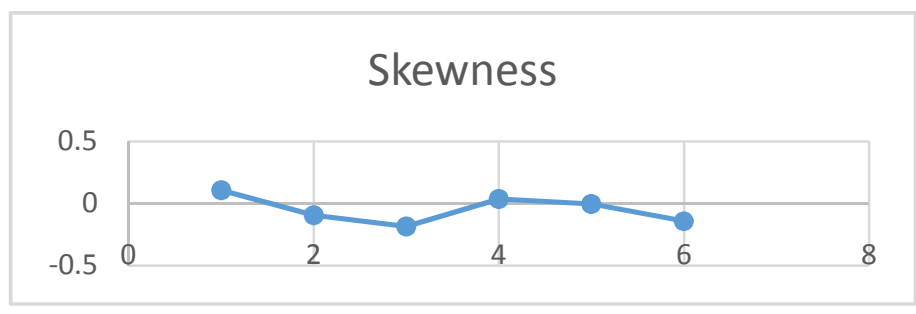

Fig. 5.Skewness

From the above analysis of the statistical parameters, the logarithmic decrement model is adopted to estimate the distances between the terminals and the Bluetooth sniffers. The RSSI values and the distances have the normal distribution relationship.

\subsection{The logarithmic decrement model}

According to the empirical characteristics of RSSI values, the RSSI presents a varying trend in logarithmic decrement. As a result, we adapt the logarithmic distance attenuation model to estimate the distances between the AP and the moving objects in order to get a more accurate result.

$$
\operatorname{RSSI}(\mathrm{d})=\operatorname{PL}\left(d_{0}\right)-10 \lg \left(\frac{d}{d_{0}}\right)-X_{\sigma}
$$

Where PL(d0) denotes the signal strength at the reference point $d 0 . n$ is the environment factor. In practical application, the environment is not the free space. There exist many different blocks. The received signal strength is not necessarily in inverse proportion of the square of the distance. As a result, the environment factor, $\mathrm{n}$, is introduced. $\mathrm{X} \sigma$ is the signal variance which describes the attenuation of the signal.

\subsection{The distance constraint}

In this study, the Bluetooth positioning results are presented in the form of three dimension. Considering the fact that the Bluetooth sniffers are installed on the roof, it is important to constrain the terminal points at some certain height. Otherwise, the positioning results may float seriously and cannot achieve the aim. The logarithmic decrement model is further analyzed to get the distance interval in which the signal strength is relatively stable. In this way, the signal strength received can be constrained in this interval.

\subsubsection{A modification based on RSSI ranging}

From the experiments, it is discovered that different environments have great influence on the spread of signals. The relationship between the distance and signal strength is not steady. So it is necessary to modify these errors. As we can see from the logarithmic decrement model, the main parameters that affect accuracy are $\mathrm{n}$ and $\operatorname{RSSI}(\mathrm{d} 0)$. 
To increase the accuracy of the model, the following modification is applied to the original model. In this modification, the RSSI values are measured every $\delta \mathrm{d}$.

$$
\operatorname{RSSI}(\mathrm{d}+\delta \mathrm{d})=\operatorname{RSSI}\left(d_{0}\right)-10 \operatorname{nlg}\left[\frac{\mathrm{d}+\delta \mathrm{d}}{d_{0}}\right]+X_{\sigma}
$$

This study makes a simulation of the modification model assigning PL(d0) $188 \mathrm{dBm}$. As shown in Figure 3-1, the environment factors are assigned 2, 1.6, 1.5 and 3.

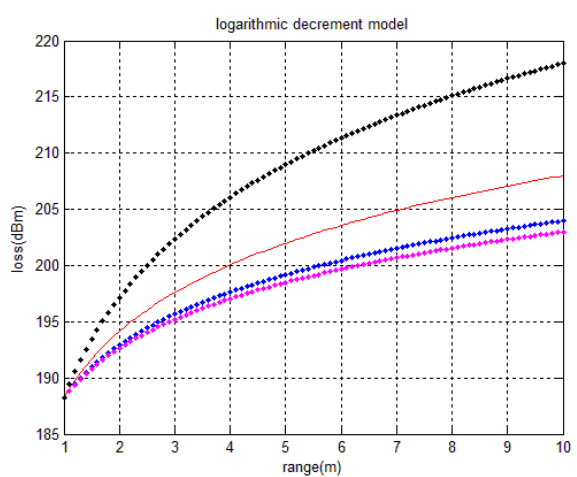

Fig. 6. Logarithmicdecrementmodel

As a result, the relatively accurate decrement factor $\mathrm{n}$ can be acquired. Combined with the modified model, we sketch the experiment results on the figure. It can be seen that the sketch line is very close to the one with $\mathrm{n}$ equaling 1.5 . After calculating related parameters, we choose 1.3 as the value of $n$.

\subsubsection{The distance constraint}

Based on the modified model, the features of the RSSI signals can be analyzed. Form the figure, it can be concluded that when the distance is larger than ten meters, the RSSI loss becomes steadily changed. This feature suggests that the distance is hard to distinguish when it is more than ten meters. So those signals can be abandoned.

Analyzing the mean values of RSSI at different distances, it presents a decreasing trend as a whole. When the distance is larger than seven meters, the signal strength cannot be sensed by the sniffer. And the signal strength is strong and stable between the interval of [2, 4]. When it comes to the signal variance, it can be seen from the chart that the fluctuation is not very serious. So the interval $[2,4]$ is chosen because of the fine fluctuation.

In conclusion, the positioning height is constrained between two meters and three meters. In this interval, the received signal strength presents reasonable statistical properties. In practical application, the Bluetooth bracelet people carry is about one meter high, so only the two-meter signal strength is the focus of our study. 


\section{Analyzing the accuracy of the ranging model}

The definition of the accuracy of positioning is the error distance between the real object position and the estimated point. The accuracy is not only determined by the RSSI accuracy between access point and terminal point, but also is related with the constraint algorithm.

For two-dimension model, the accuracy can be defined as:

$$
\sqrt{(\Delta x)^{2}+(\Delta y)^{2}}
$$

For three-dimension model, the position accuracy is defined as:

$$
\sqrt{(\Delta x)^{2}+(\Delta y)^{2}+(\Delta z)^{2}}
$$

Where is equal to the height of the moving object. For convenience, this study only analyzes the precision of the position on the vertical direction. The following table shows the measured RSSI values and the estimated values using the modified model. And the errors are also given in the table.

\begin{tabular}{|c|c|c|}
\hline estimate value & measured value & relative error \\
\hline 179.1114772 & 188.4432 & $4.95 \%$ \\
\hline 179.4422756 & 179.4606 & $0.01 \%$ \\
\hline 181.0740356 & 173.5975 & $0.11 \%$ \\
\hline 177.9528233 & 181.2825 & $2.51 \%$ \\
\hline 174.9449357 & 173.194 & $1.01 \%$ \\
\hline 177.6542791 & 175.0726 & $1.47 \%$ \\
\hline
\end{tabular}

Fig. 7. Comparisonwithresults

By comparing the results of data we found that our model is accurate and improve the accuracy of ranging ${ }^{[6,7]}$, which will further improve the accuracy of positioning. For example, at two meters and three meters when our relative error is only $0.1 \%$ and 0.01 .It can meet the positioning requirements very well.

\section{Conclusion}

In this paper, we study the constraints to the model based on RSSI ranging location. In the experimental environment, our key research is the signal strength which receive from the Bluetooth sensor network. According to the signal data, we analyze the statistical characteristics of the signal data, such as the mean value and the signal variance of the signal. Finally, we obtain the relation model between distance and signal intensity. Then the model is validated by the experiment. We found that the model has a better constraint effect on the instability and inaccurate of Bluetooth positioning. Generally, the constraint algorithm in this paper can be further studied. For example, considering the influence of environment, such as temperature and humidity, considering the influence of many Bluetooth devices to locate simultaneously and so on. 


\section{References}

[1] Indoor Location Algorithms Design and Implementation[D].Beijing: Beijing University Of Posts And Telecommunications, 2010.16-41.

[2]Shusheng Liu. Application Of Bluetooth Technology [M] . Shenyang: Northeast University Press, 2001.

[3]Gang Zhu. The Principle and Protcol on Bluetooh Technology [M]. Beijing: Beijing Jiaotong University Press, 2002.

[4]Lulin Zhang. The Application and Protcol on Bluetooh Technology [M] . Beijing: Posts \& Telecom Press, 2001.

[5]Zhifeng Zhao ， Shaoren Zheng.Ad Hoc Research of Network Structure，Telecommunications Science, 2001, 17.

[6]Xin Shi, Aimin Yin, Xi Chen. RISS and multidimensional scalling based indoor localization algorithm[J]. Chinese Journal Of Scientific Instrument, 2014, 35(2):261-268.

[7]Xin Yuan, Xiaoping Wu, Guoyin Wang. Accurate Computation Approach of RSSI-Based Localization with Linear Least Square Method[J]. Chinese Journal Of SENSORS AND ACTUATORS, 2014(10):1412-1417.

[8]Pei L, Chen R, Liu J, et al. Using Inquiry-Based Bluetooth RSSI Probability Distributions for Indoor Positioning[J]. 2010, 9:122-130122.

[9] Janja Svečko, Marko Malajner, Dušan Gleich . Distance estimation using RSSI and particle filter. 《Isa Transactions》, 2014, 55(16):7146-7158

[10] Oguchi K, Shou M, Dai H. Human Positioning Estimation Method Using Received Signal Strength Indicator (RSSI) in a Wireless Sensor Network [J]. Procedia Computer Science, 2014, 34(7):126-132.

[11] Pei L, Chen R, Liu J, et al. Inquiry-Based Bluetooth Indoor Positioning via RSSI Probability Distributions[C] International Conference on Advances in Satellite \& Space Communications. IEEE Computer Society, 2010:151-156.

[12] Wang Y, Yang X, Zhao Y, et al. Bluetooth positioning using RSSI and triangulation methods[C] Consumer Communications and NETWORKING Conference. 2013:837-842.

[13] Kajioka S, Mori T, Uchiya T, et al. Experiment of indoor position presumption based on RSSI of Bluetooth LE beacon[C] Consumer Electronics. IEEE, 2015:14. 